
Not for reproduction, distribution or commercial use.
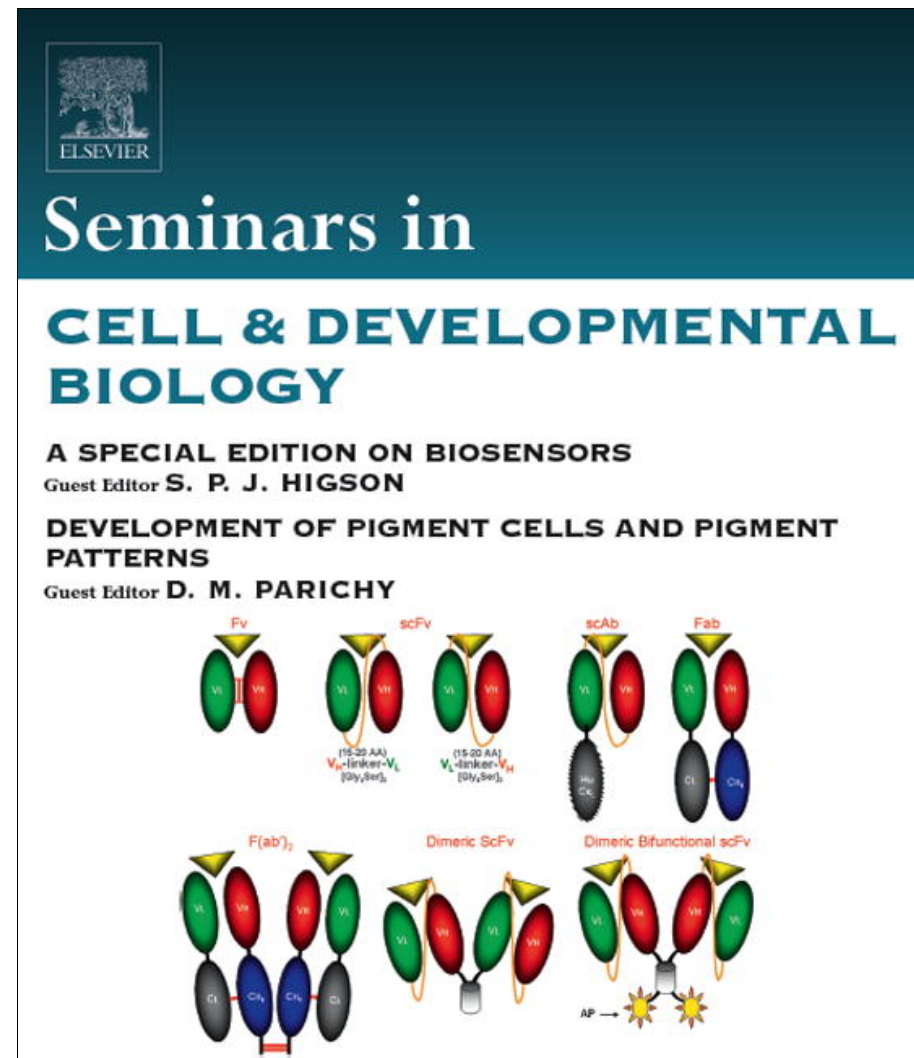

Volume 20, issue 1, February 2009
ISSN $1084-9521$

This article appeared in a journal published by Elsevier. The attached copy is furnished to the author for internal non-commercial research and education use, including for instruction at the authors institution and sharing with colleagues.

Other uses, including reproduction and distribution, or selling or licensing copies, or posting to personal, institutional or third party websites are prohibited.

In most cases authors are permitted to post their version of the article (e.g. in Word or Tex form) to their personal website or institutional repository. Authors requiring further information regarding Elsevier's archiving and manuscript policies are encouraged to visit: 
Review

\title{
Development and evolution of insect pigmentation: Genetic mechanisms and the potential consequences of pleiotropy
}

\author{
Patricia J. Wittkopp ${ }^{a}$, Patrícia Beldade ${ }^{\text {b,c,* }}$ \\ a 830 North University Avenue, Department of Ecology and Evolutionary Biology, Department of Molecular, Cellular, and Developmental Biology, \\ University of Michigan, Ann Arbor, MI 48109-1048, USA \\ ${ }^{\mathrm{b}}$ Institute of Biology of Leiden University (IBL), PO Box 9516, 2300 RA, Leiden, The Netherlands \\ ${ }^{\mathrm{c}}$ Instituto Gulbenkian de Ciência (IGC), Apartado 14, P-2781-901 Oeiras, Portugal
}

\section{A R T I C L E I N F O}

\section{Article history:}

Available online 14 October 2008

\section{Keywords:}

Drosophila

Lepidoptera

Melanization

cis-regulation

Variation

\begin{abstract}
A B S T R A C T
Insect pigmentation is a premier model system in evolutionary and developmental biology. It has been at the heart of classical studies as well as recent breakthroughs. In insects, pigments are produced by epidermal cells through a developmental process that includes pigment patterning and synthesis. Many aspects of this process also impact other phenotypes, including behavior and immunity. This review discusses recent work on the development and evolution of insect pigmentation, with a focus on pleiotropy and its effects on color pattern diversification.
\end{abstract}

(c) 2009 Published by Elsevier Ltd.

\section{Contents}

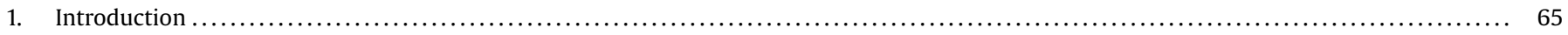

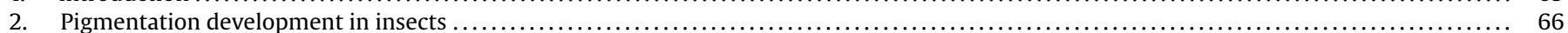

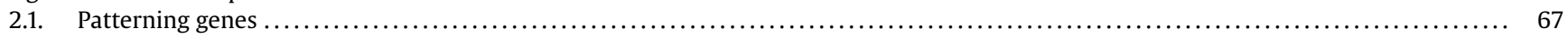

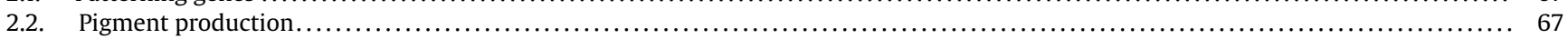

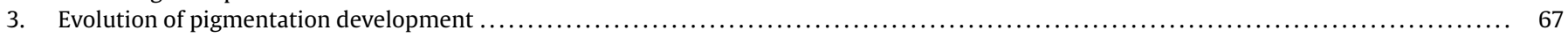

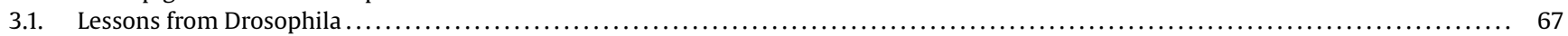

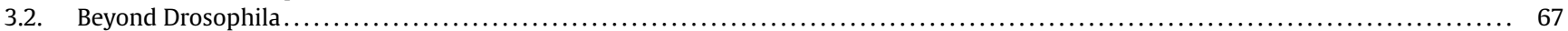

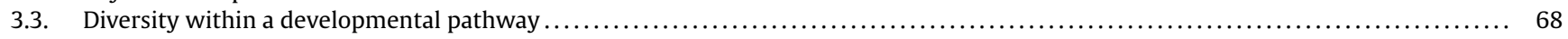

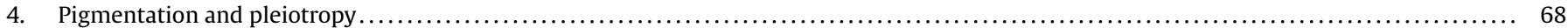

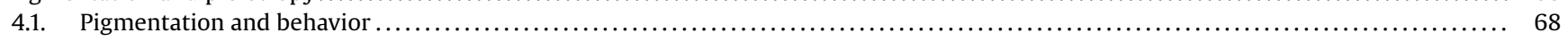

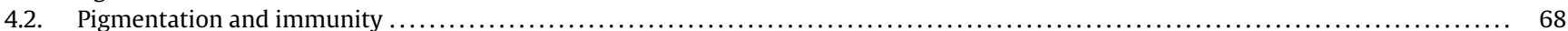

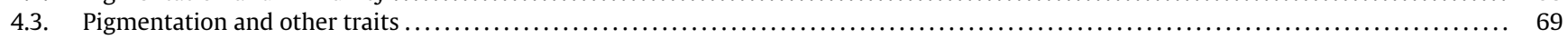

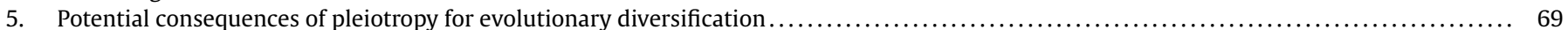

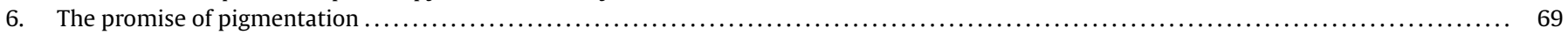

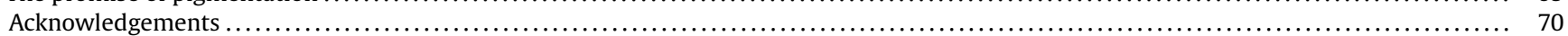

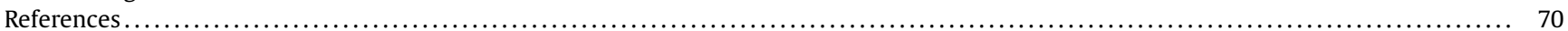

\section{Introduction}

The extraordinary diversity of insect colors and color patterns has long fascinated biologists and laymen alike. It inspires collectors, students and the general public, and it is a powerful tool for

\footnotetext{
* Corresponding author. Tel.: +31 71 5274853; fax: +31 715274900 .

E-mail addresses: wittkopp@umich.edu (P.J. Wittkopp), p.beldade@biology.leidenuniv.nl (P. Beldade)
}

stimulating interest in science education. For specialists, studies of insect pigmentation have provided insight into diverse branches of biology, including ecology, development, genetics, and physiology. More recently, studies of insect pigmentation have taken center stage in the young field of evolutionary developmental biology, contributing to emerging principles such as the co-option of shared genetic circuitry for the evolution of novel traits (examples in butterfly wing patterns [1-3]) and the prevalence of cis-regulatory evolution in morphological diversification (examples in Drosophila pigmentation [4-7]). 
(a)

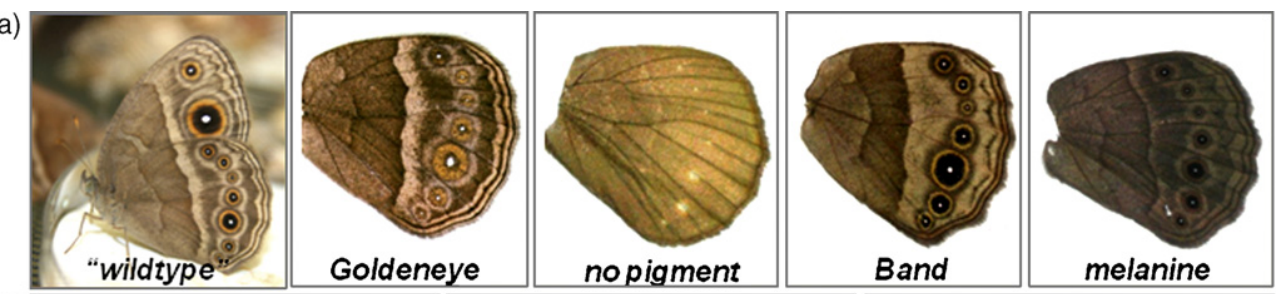

(b)

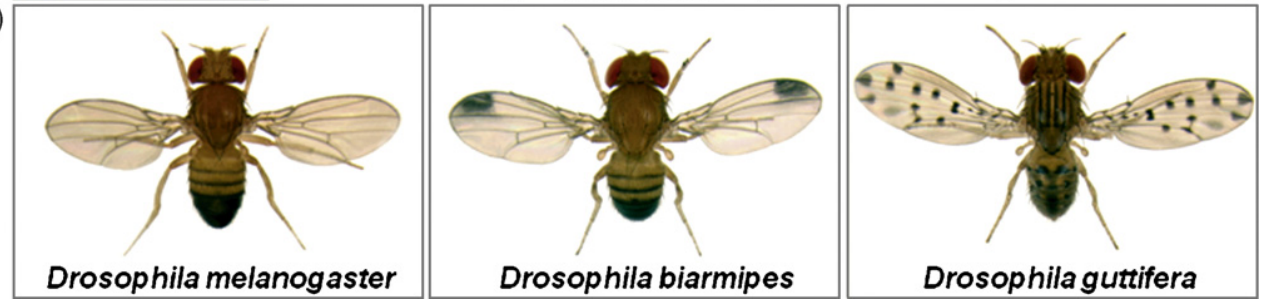

Fig. 1. Variable pigmentation in lab-tractable systems. Examples of intraspecific and interspecific variation in two insect orders are shown. (a) Variable wing color in spontaneous laboratory mutants of Bicyclus anynana butterflies (photos courtesy of Suzanne Saenko). Note the changes in both pigment colors and locations. (b) Interspecific diversity in Drosophila pigmentation (photos courtesy of Nicolas Gompel and Benjamin Prud'homme). Note the differences in wing, thorax, and abdominal pigmentation among species.

Insect pigmentation is a highly variable trait, with spectacular differences between species, between populations of the same species, and between individuals within a population. Pigmentation also varies between life stages of single individuals and between body parts of individual life stages. Diversity exists in terms of which colors are used as well as how these colors are arranged into patterns. The ecological pressures that promote this diversity are themselves diverse, with no single adaptive role explaining variation in all species. Pigmentation functions in visual communication, either between members of the same species (e.g. intraspecific recognition and mate choice) or different species (e.g. mimicry, aposematism, deflection, and camouflage used to avoid predation), and in physiological processes such as thermoregulation, photo-protection, and desiccation resistance (examples in [8]).

The combination of phenotypic diversity and laboratory mutants (Fig. 1), adaptive roles in ecology and evolution, and a good understanding of genetics and development make insect pigmentation well-suited for investigating the reciprocal interactions between evolutionary and developmental processes that shape phenotypic variation. Furthermore, the links between pigmentation and other phenotypes provide an opportunity to examine the genetic mechanisms and evolutionary consequences of pleiotropy. Here, we (1) review the mechanisms of pigmentation development in insects, (2) illustrate how development differs within and between species, (3) highlight features of pigmentation development that provide opportunities for pleiotropy, and (4) discuss how pleiotropy may influence the evolution of pigmentation and/or other traits. We close by highlighting some future directions for studies of insect pigmentation in evolution and development.

\section{Pigmentation development in insects}

Unlike vertebrates, who use specialized cell types and cell migration to create body pigment patterns (reviewed in [9]), insects typically synthesize body color pigments and/or pigment precursors in their epidermal cells. In some cases, pigments are found within modified epidermal cells (e.g. scales on butterfly wings [10]), but typically these molecules are incorporated into the hard exoskeleton overlying the epidermal cells through a process known as sclerotization [11]. Thus, insect body color is not simply painting on a wall; but rather, it is like the color in true frescos-an integral part of the wall itself. (Note that this does not apply to eye pigmentation, where pigments are localized to a specific cell type.)
Among insects, pigmentation development is best understood in D. melanogaster. Pigmentation is one of few adult traits in this species for which regulatory genes affecting developmental patterning, genes ultimately producing the adult trait, and biochemical links between them are known in detail. Our discussion of pigmentation development and evolution exploits this knowledge, dividing the pigmentation process into two stages (Fig. 2): (1) positioning pigments in space and time and (2) the biochemical synthesis of pigments. Genes involved in these two steps will be referred to as "patterning" and "effector" genes, respectively. Patterning genes regulate the distribution of pigments by directly or indirectly activating expression of effector genes that encode the enzymes and co-factors required for pigment biosynthesis.
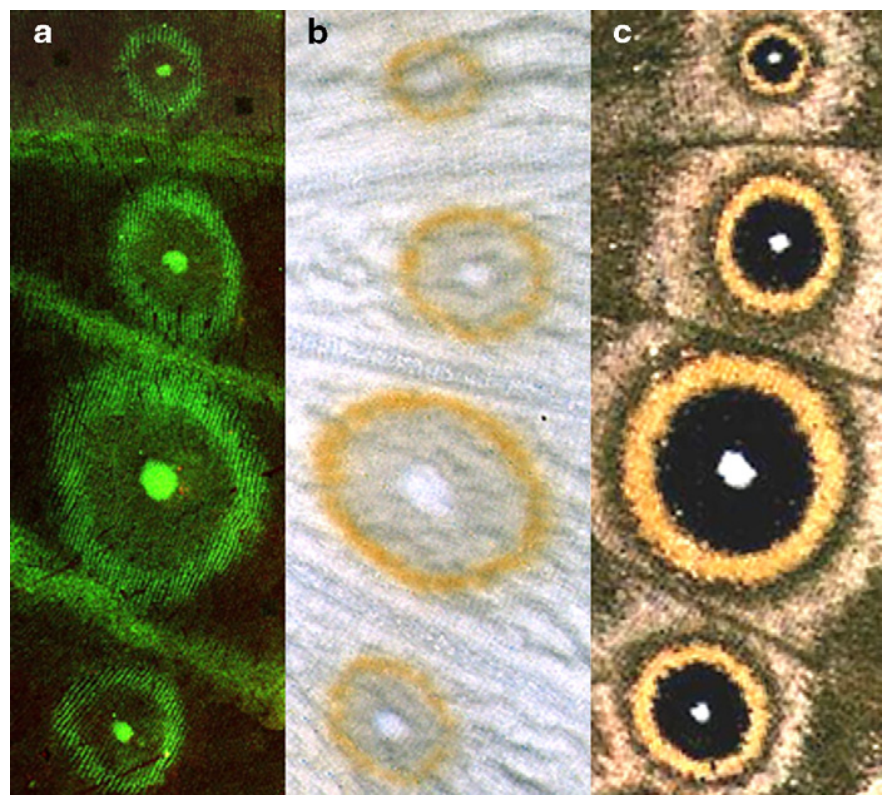

Fig. 2. Stages of pigmentation development. During the formation of eyespots on the hindwing of Bicyclus anynana butterflies, Engrailed protein (green) is found in early pupal scale-forming cells ((a) photo by Suzanne Saenko). These cells will later synthesize the yellow pigment ((b) photo by Nicolien Pul) that makes up the golden ring of adult eyespots (c). After "patterning" genes such as Engrailed specify the location of pigments, "effector" genes, which encode the enzymes responsible for pigment production, determine the nature and quantity of pigments produced. 


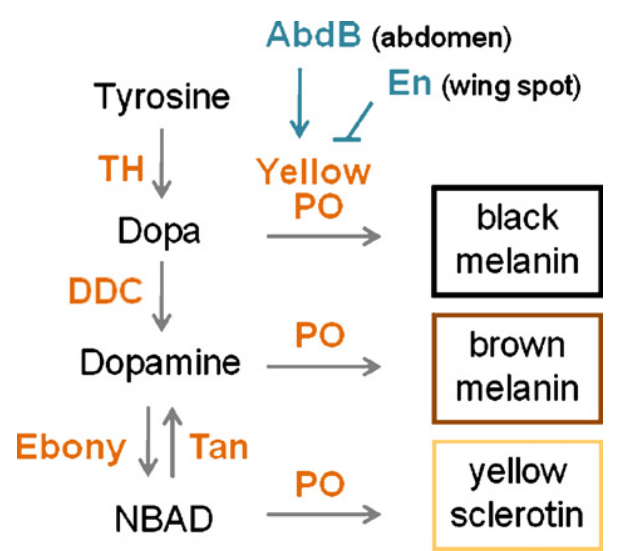

Fig. 3. Melanin synthesis in Drosophila. Central components of the melanin synthesis pathway are shown, with enzymes indicated in orange and pigments as well as pigment precursors indicated in black. Different cells express different components of this pathway, and thus produce different pigments in the overlaying adult cuticle (more details in [58]). Shown in blue are two examples of direct regulators controlling expression of the yellow gene. The Engrailed (En) protein binds to a cis-regulatory sequence controlling expression in the wing "spot" of $D$. biarmipes, repressing its expression [4]; and the Abdominal-B protein (AbdB) binds to a cisregulatory sequence controlling male-specific pigmentation in the $D$. melanogaster abdomen, activating its expression [98] (TH, Tyrosine Hydroxylase, DDC, Dopadecarboxylase, PO, phenoloxidases, NBAD, N-beta-alanyl-dopamine)

\subsection{Patterning genes}

In $D$. melanogaster, pigment patterning is controlled by pleiotropic regulatory proteins, including sex determination genes (e.g. doublesex), HOX genes (e.g. Abdominal-B), signaling pathways (e.g. wingless and decapentaplegic), and selector genes (e.g. optomotor-blind, bric-a-brac and engrailed) (reviewed in [12]). These factors regulate pigmentation as well as multiple other traits. Additional pleiotropic transcription factors are required for the development of pigmentation in other insect lineages. For example, the Ultrabithorax gene, required for haltere specification in dipterans, discriminates between forewing and hindwing pigmentation in butterflies [13], and the Distal-less gene, required for insect appendage development, specifies butterfly wing color pattern elements [14]. Such reuse of regulatory proteins for diverse functions is a general theme in development and evolution [15].

\subsection{Pigment production}

Effector genes compose multiple pigment biosynthesis pathways, with each pathway producing a distinct class of pigments. These genes are less pleiotropic than the transcriptional regulators controlling patterning, although they still affect multiple phenotypes (see Section 4). Melanins, a predominant class of insect pigments, are synthesized by a branched biochemical pathway that converts phenlyalanine into tyrosine and then polymerizes modified molecules such as dopa, dopamine, and N-beta-analyl-dopamine into black, brown, and yellow pigments (Fig. 3). Spatiotemporally regulated expression of the yellow, tan and ebony effector genes determines the location and relative abundance of these three pigments $[5,16]$. The biochemical pathway leading to the production of ommochromes (red, brown, and yellow pigments) includes the cinnabar, vermilion, and white genes and is also widely used among insects. Those pigments are synthesized from tryptophan and packaged into cytosolic pigment granules. They are limited to eye color in Drosophila, but contribute to wing pigmentation in other species [17,18]. Similarly, pteridines (red, yellow, orange), produced by a pathway including the rosy and purple genes, are also restricted to eye color in Drosophila yet play additional roles in other insects [19]. Other pigments found in insects include carotenoids (orange-yellow) and flavonoids (bluish hues), which are extracted from food and used with little modification, and more specialized pigments such as anthraquinones (violet, blue, green) in Coccidae, aphins (purplish-red) in Aphididae, and papilliochromes in some butterflies [20,21]. Links between pigment biosynthesis pathways have also been reported $[21,22]$.

\section{Evolution of pigmentation development}

Pigmentation diversity indicates that mechanisms of pigmentation development are not static, but rather vary among and within species. Genetic analysis of these differences provides some of the most detailed case studies of evolutionary change. These studies also provide a rare opportunity to investigate how intraspecific variation and interspecific divergence are structured within a developmental pathway. Below, we review recent studies examining the genetic basis of pigmentation differences.

\subsection{Lessons from Drosophila}

The most detailed evolutionary genetic work examines pigmentation differences within and among Drosophila species. Within $D$. melanogaster, genetic mapping suggests that functional variants affecting pigmentation lie within both transcriptional regulators (bric-a-brac [23], optomotor-blind [24]) and pigment synthesis genes (ebony [25]). Between Drosophila species, genetic mapping suggests functional divergence at loci encoding pigment synthesis effector genes (ebony [26], tan [5,27]), although these studies do not exclude a role for patterning genes. Both patterning (bric-a-brac [28]) and effector (yellow[29], ebony [26], tan [5]) genes have expression differences that correlate with pigmentation divergence between species. For three of these four genes (bric-a-brac [7], yellow [4], and tan [5]), functional cis-regulatory changes have been identified, with changes in bric-a-brac and yellow shown to alter binding sites for highly pleiotropic transcriptional regulators (Doublesex [7], Abdominal-B [5,7], and Engrailed [4]). For ebony, cis-regulatory changes have not been definitively proven, but data are consistent with cis-regulatory divergence [26].

\subsection{Beyond Drosophila}

Studies of pigmentation development in D. melanogaster provide candidate genes that may contribute to pigmentation diversity in other insect lineages. Such an approach has been applied most extensively to the diverse wing patterns of butterflies. Comparing spatial patterns of gene expression among species has shown that differences in engrailed, spalt and Distal-less expression are associated with the diversification of color rings in butterfly eyespots [30]. These expression differences could be caused by cis-regulatory changes in the genes examined or by changes in their transcriptional activators. Although specific genetic changes have not yet been identified, studies mapping the genetic basis of variable wing pigmentation are consistent with changes at pleiotropic regulatory genes: Distal-less is associated with quantitative variation in eyespot size in Bicyclus anynana [31], wingless is associated with wing color in hybrids of Heliconius species [32], and engrailed/invected is associated with wing pattern polymorphism in Papilio dardanus [33]. Increased mapping resolution and/or functional analyses are ultimately needed to separate the effects of these candidate genes from linked loci. 
The contribution of pigment synthesis genes to phenotypic diversity is also starting to be explored in different butterfly species. Genes from the ommochrome pathway (white, vermilion, Henna, and cinnabar) are expressed in developing butterfly wings in patterns correlating with the adult pigmentation $[18,34]$. Similarly, expression of genes in the melanin synthesis pathway $(T H, D d c$, yellow, and ebony) correlates with larval cuticle pigmentation development in Papilio [35-37]. Orthologs of pigmentation effector genes have been identified in a number of other insects (e.g. [38-40]), and the mutant phenotypes analyzed thus far suggest that their roles in pigmentation development are conserved [41,42]. It remains to be seen how often genes identified through mutant analysis contribute to the genetic basis of evolutionary change [43].

\subsection{Diversity within a developmental pathway}

A paradigm is emerging for the genetic basis of pigmentation evolution that includes frequent changes in the identity of pleiotropic transcription factors regulating expression of pigmentation genes. These changes are mediated by the gain and loss of transcription factor binding sites in the cis-regulatory regions of these genes [5-7]. Changing the binding sites for patterning factors in cis-regulatory regions of more specialized effector genes is assumed to be less pleiotropic than changing the transcription factor genes themselves. This is because of the modular architecture of cis-regulatory regions, which have discrete elements controlling transcription in different spatiotemporal domains [44-47]. However, cis-regulatory changes in patterning genes encoding transcription factors can still affect expression of the transcription factor's target genes. Such is the case for cis-regulatory changes in the bric-a-brac gene; changing expression of bric-a-brac in the D. melanogaster abdomen alters pigmentation as well as the distribution of bristles and trichomes [28]. In general, cis-regulatory changes have reduced pleiotropy relative to changes in coding sequences, but it is important to remember that they do not necessarily eliminate pleiotropy completely.

\section{Pigmentation and pleiotropy}

Pleiotropy (i.e. a single gene or nucleotide affecting multiple traits) is often invoked as a mechanism to explain covariance among traits, but it can be difficult to prove. The strongest evidence for pleiotropy comes from phenotypic analysis of genotypes with single mutations. If a mutation alters more than one phenotype, it is said to be pleiotropic. Outside of genetic model systems, where mutational analyses are often not feasible, circumstantial evidence of pleiotropy is derived from correlated phenotypes in artificial selection experiments, natural populations, or across phylogenetic trees. While these correlations can indeed result from pleiotropy, they can also be caused by genetic linkage, shared environmental conditions, or correlated selection pressures. In insects, associations with pigmentation have been observed for behavior, immunity, life-history, physiological, and developmental traits. The following sections provide a sampling of these pleiotropic effects, with a focus on Drosophila melanogenesis, which offers the most unequivocal evidence of pleiotropy and the most complete understanding of the molecular mechanisms underlying it (see insightful review by John True [8]).

\subsection{Pigmentation and behavior}

An association with pigmentation has been described for diverse behaviors, including swarming [48], feeding [49], social dominance [50], mate preference [32], and courtship display [51]. The molecular basis for these associations, as well as the data supporting them, are also varied. For example, in Schistocerca gregaria grasshoppers the alternative color/behavior morphs are the product of phenotypic plasticity [52,53], and the association between the two suggests shared components in their underlying developmental and/or physiological mechanisms. In Tetrix undulate pigmy grasshoppers, behavior and pigmentation both seem to be genetically determined [49], but there is no clear evidence of pleiotropy, suggesting correlated selection pressures. In Heliconius butterflies, genetic mapping has shown co-segregation of wing color and mate preference phenotypes in interspecific crosses [32], but the mapping resolution was insufficient to discriminate between pleiotropy and genetic linkage. In Drosophila, genetic analysis of wing pigmentation and courtship display indicates a role for both linkage and pleiotropy in the co-evolution of these traits [51].

The clearest evidence for pleiotropy between insect pigmentation and behavior comes from studies of pigmentation mutants in D. melanogaster. The genes tan, ebony, Dopa-decarboxylase and yellow all affect both body color and behavior [54]. These genes are expressed in epidermal cells, where they are used to synthesize pigments (Fig. 3), as well as in neurons and neuron-associated cells, where they putatively influence behavior [55-57]. The Tan protein catalyzes the conversion of $\mathrm{N}$-beta-analyl-dopamine to dopamine during melanin production, and of carcinine to histamine during neurotransmission [58]. Ebony catalyzes the reverse hydrolysis reaction, suggesting it is playing a complementary role to Tan in the cycling of neurotransmitters [57]. The enzyme Dopa-decarboxylase functions upstream of Ebony and Tan in dopamine synthesis, and is required for the production of both histamine and serotonin neurotransmitters [59]. The yellow gene is upregulated in malespecific neurons, consistent with its requirement for normal male courtship behavior $[56,60]$. Links between pigmentation and neurobiology may even extend beyond melanin-related molecules: ommochrome-containing pigment granules have been found in the central nervous system of Bombyx mori, although their function there, if any, is not known [61].

\subsection{Pigmentation and immunity}

Another phenotype often associated with pigmentation in insects is immunity. This includes innate immunity, wound healing, and parasite defense. The presence of a brown-black pigment accompanying cellular innate immunity is a unique characteristic of arthropods [62] and can be used as an indicator of immune investment [63]. During wound healing in Drosophila, melanization serves as a "scab" to protect the epidermal opening during cellular repair [64]. In butterflies, epidermal wounding can induce the formation of pigments other than black-brown melanin [65], but the purpose and underlying mechanism of this induction are unknown. As structural components of the insect cuticle, pigments contribute to the physical barrier that protects the insect body [66]. When this barrier is penetrated, melanization is used to encapsulate the foreign object and prevent infection [67]. In natural populations, increased melanization improves the physical barrier against infection and/or the rate of parasite encapsulation (e.g. $[68,69])$. It has even been speculated that the melanin synthesis pathway functions as a mechanism for parasite recognition [62].

Genetic analysis of $D$. melanogaster mutants again provides strong evidence of pleiotropy between body color and immunity. Mutations in pale (encoding Tyrosine Hydroxylase) and Dopa-decarboxylase affect pigmentation and the melanic immune response [59,62,70,71]; and genes encoding phenoloxidases polymerize dopa and dopamine into melanins during pigmentation development as well as wound healing and encapsulation [69,72-75]. Despite the production of similar pigments, melaniza- 
tion that occurs as part of the immune response and melanization that is used for body pigmentation, does not require all of the same genes. For example, the yellow gene, which is strictly required for the production of black melanin in the developing cuticle, is not necessary for the formation of a black melanin scab in a wounded fly (Wittkopp, unpublished observation).

\subsection{Pigmentation and other traits}

Many other aspects of pigmentation and pigmentation development can also cause pleiotropy. In some cases, this pleiotropy results from properties of the pigments themselves. One example is desiccation resistance, in which melanin affects the permeability of the cuticle [76]; Drosophila with greater melanization are more resistant to desiccation $[77,78]$. Co-factors shared by multiple processes also result in pleiotropy. For example, mutations in a gene required for copper uptake (DmATP7) show defects in early embryonic development, larval growth, and adult pigmentation [79], with the pigmentation phenotypes resulting from disruption of copper-dependant phenoloxidase activity and the other phenotypes resulting from compromising other cuproenzymes. Intermediates produced during pigment synthesis are yet another source of pleiotropy. In addition to pigmentation and neuronal function, dopamine is required for cell migration in the developing trachea [80]. Even life-history traits such as longevity are affected by pleiotropic pigmentation genes. In $D$. melanogaster, single nucleotide polymorphisms associated with variation in longevity have been identified within the Dopa-decarboxylase [81] and catsup [82] genes. (Catsup is a negative regulator of Tyrosine Hydroxylase.) An effect on lifespan has also been described for genes involved in pteridine synthesis [83]. Descriptions of mutant phenotypes in FlyBase, the online collection of data from Drosophila genetics [54], provide many more examples of pleiotropy for pigmentation genes. (When evaluating the extent of pleiotropy, it is important to note that the definition of a phenotype is subjective. What researchers may describe as two different traits may not be caused by distinct biological processes. For example, tan mutants show disrupted histamine levels and photoreceptor function [58]-presumably, one disruption causes the other.)

\section{Potential consequences of pleiotropy for evolutionary diversification}

At first glance, pleiotropy is expected to impact adaptation by increasing the likelihood that a mutation altering pigmentation will affect other phenotypes. However, the great diversity in insect pigmentation argues against strong constraints and suggests that at least some of the potential limitations imposed by pleiotropy can be overcome. As discussed in Section 3.3, one way in which a pleiotropic gene can subdivide its functions is through modular cisregulation. Most genes have multiple, independent cis-regulatory elements that control spatial and temporal gene expression; mutations in one element can have no effect on functions mediated by the other element [44-47]. A similar situation occurs within coding regions when the protein has trait-specific domains [84]. Rather than constraining overall pigmentation divergence, pleiotropy may primarily bias the specific genetic changes by which pigmentation evolves. In other words, pleiotropy may have a larger effect on the mode than on the extent of phenotypic diversification.

Despite the presence of discrete functional regions within a pleiotropic gene, the close physical proximity of these regions can still limit their independent evolution-especially over short timescales. Genetic linkage causes nearby regions to be inherited together more often than expected by chance, and many meioses are needed for sufficient recombination to "separate" two closely linked sites. In natural populations of $D$. melanogaster, linkage disequilibrium typically extends a few hundred nucleotides [85], suggesting that functional modules located close to each other can be inherited together for long periods of time. (The specific extent of linkage disequilibrium varies greatly across the genome and among species, and is affected by selection, recombination rate, and demographic parameters.) In a gene such as yellow, which has separate cis-regulatory elements affecting adult body pigmentation and behavior that are located ca. 400 bp apart [60], a new mutation affecting one trait will often be transmitted with the linked allele affecting the other trait. With sufficient time and/or large enough population sizes, variants in these different modules can be inherited independently, allowing for uncorrelated evolution of the traits. However, over shorter evolutionary timescales and especially in smaller populations, physical linkage between neighboring mutations affecting different cis-regulatory elements can cause correlated changes among phenotypes. In this way, pleiotropic effects of alleles may persist despite independent functional regions.

An additional challenge for thinking about the effects of pleiotropy on evolution is that pleiotropy is ultimately a property of alleles rather than genes. Loss-of-function alleles that significantly disrupt (or completely eliminate) a gene's activity are typically used in the laboratory to determine the gene's function and assess pleiotropy, but such extreme mutations are rarely found in natural populations. Alleles segregating in the wild tend to have more moderate effects. For example, mutant phenotypes indicate that ebony, bric-a-brac, and optomotor-blind are all pleiotropic genes, yet they exhibit variation in natural populations that correlates with pigmentation diversity [23-25]. Studies of sequence variation in catsup show precisely how pleiotropy can be subdivided by single nucleotide polymorphisms [82]. A recent analysis of quantitative trait loci in mice is also consistent with this idea, showing that individual alleles observed in quantitative genetic mapping studies are less pleiotropic than was indicated by developmental genetic analysis [86]. Taken together, these studies suggest that mutations that somehow subvert a gene's pleiotropic effects may be over-represented in natural populations.

\section{The promise of pigmentation}

As shown in this review, insect pigmentation is a bourgeoning area of research; one that has already provided critical insights into the developmental and genetic mechanisms of phenotypic evolution. Despite these contributions, many questions remain. Of these, perhaps the most pressing are "Do the findings from Drosophila hold for other species?" and "Will extending the color pallet lead researchers to paint a different picture of pigmentation evolution?" Detailed genetic analysis of natural variation in other insects and in other pigment pathways will address these questions. Such studies will reveal, for example, whether the predominant role of cisregulatory evolution observed for Drosophila melanin patterning is a general feature of insect pigmentation divergence. Studies of morphological traits controlled by pleiotropic developmental pathways in a variety of organisms suggest cis-regulatory changes are indeed a pervasive source of phenotypic diversification (examples in [87-90]; recent reviews $[45,46]$ ), while studies of variation in traits controlled by developmental systems with less pleiotropy such as melanin synthesis activated by the dedicated Melanocortin 1 receptor in specialized vertebrate cells - suggest changes in amino acid sequence can be common as well [91].

With genetic and genomic analysis now feasible outside of classical model organisms [92-94], researchers can begin capitalizing on what makes pigmentation such a unique system: its astounding diversity. Growing collections of genes in diverse groups of 
insects allow a less "Drosophila-centric" investigation of this diversity, which is critical for determining the role of lineage-restricted genes and developmental processes in phenotypic evolution.

Pigmentation is also well-suited for merging evolutionary developmental biology studies of interspecific divergence with quantitative genetic analysis of intraspecific variation. An integration between these two (often disparate) fields is essential for understanding the relationship between variation within species and divergence between species [47,95]. Finally, intricate color patterns are a model for complex traits because they require the activity of many genes and interactions between them. Geneby-environment interactions - an important, yet under-studied determinant of complex traits - can also be examined in this system: developmental plasticity for insect pigmentation is common (e.g. $[7,10])$, and some mechanisms by which environmental cues affect pigmentation have been identified $[96,97]$.

In summary, insect pigmentation holds great promise for linking variation in genes to variation in development, variation in development to variation in phenotypes, and variation in phenotypes to variation in fitness, which is necessary for a comprehensive understanding of evolutionary diversification and the genetics of adaptation.

\section{Acknowledgements}

We thank David Parichy for the joint assignment and organization of the present volume; Nicolas Gompel, Benjamin Prud'homme, Suzanne Saenko, and Nicolien Pul for sharing pictures included in Figs. 1 and 2; Tony Long and Peter Andolfatto for helpful discussion; and Artyom Kopp for thoughtful comments on the manuscript. PB is supported by grants from the Dutch Science Foundation NWO (VENI 863.04.013), and the Portuguese Foundation for Science and Technology FCT (PTDC/BIA-BDE/65295/2006). PJW is supported by the National Science Foundation (DEB-0640485), March of Dimes (5-FY07-181) and the Alfred P. Sloan Foundation.

\section{References}

[1] Keys DN, Lewis DL, Selegue JE, et al. Recruitment of a hedgehog regulatory circui in butterfly eyespot evolution. Science 1999;283:532-4.

[2] Monteiro A, Glaser G, Stockslager S, Glansdorp N, Ramos D. Comparative insights into questions of lepidopteran wing pattern homology. BMC Dev Biol 2006;6:52.

[3] Saenko SV, French V, Brakefield PM, Beldade P. Conserved developmental processes and the formation of evolutionary novelties: examples from butterfly wings. Philos Trans R Soc Lond B Biol Sci 2008;363:1549-55.

[4] Gompel N, Prud'homme B, Wittkopp PJ, Kassner VA, Carroll SB. Chance caught on the wing: cis-regulatory evolution and the origin of pigment patterns in Drosophila. Nature 2005;433:481-7.

[5] Jeong S, Rebeiz M, Andolfatto P, Werner T, True J, Carroll SB. The evolution of gene regulation underlies a morphological difference between two Drosophila sister species. Cell 2008;132:783-93.

[6] Prud'homme B, Gompel N, Rokas A, et al. Repeated morphological evolution through cis-regulatory changes in a pleiotropic gene. Nature 2006;440:1050-3.

[7] Williams TM, Selegue JE, Werner T, Gompel N, Kopp A, Carroll SB. The regulation and evolution of a genetic switch controlling sexually dimorphic traits in Drosophila. Cell 2008;134:610-23.

[8] True JR. Insect melanism: the molecules matter. Trends Ecol Evo 2003;18:640-7.

[9] Hoekstra HE. Genetics, development and evolution of adaptive pigmentation in vertebrates. Heredity 2006;97:222-34

[10] Nijhout HF. The Development and Evolution of Butterfly Wing Patterns. Washington: Smithsonian Inst. Press; 1991.

[11] Hopkins TL, Kramer KJ. Insect cuticle sclerotization. Annu Rev Entomol 1992;37:273-302.

[12] Wittkopp PJ, Carroll SB, Kopp A. Evolution in black and white: genetic control of pigment patterns in Drosophila. Trends Genet 2003;19:495-504.

[13] Weatherbee SD, Nijhout HF, Grunert LW, et al. Ultrabithorax function in butterfly wings and the evolution of insect wing patterns. Curr Biol 1999;9:109-15.

[14] Brakefield PM, Gates J, Keys D, et al. Development, plasticity and evolution of butterfly wing patterns. Nature 1996;384:236-42.

[15] Carroll SB, Grenier JK, Weatherbee SD. From DNA to Diversity: Molecular Genetics and the Evolution of Animal Design. Blackwell Synergy; 2001.
[16] Wittkopp PJ, True JR, Carroll SB. Reciprocal functions of the Drosophila Yellow and Ebony proteins in the development and evolution of pigment patterns. Development 2002;129:1849-58.

[17] Nijhout HF. Ommochrome pigmentation of the linea and rosa seasonal forms of Precis coenia (Lepidoptera: Nymphalidae). Arch Insect Biochem Physio 1997;36:215-22.

[18] Reed RD, Nagy LM. Evolutionary redeployment of a biosynthetic module: expression of eye pigment genes vermilion, cinnabar, and white in butterfly wing development. Evol Dev 2005;7:301-11.

[19] Morehouse NI, Vukusic P, Rutowski R. Pterin pigment granules are responsible for both broadband light scattering and wavelength selective absorption in the wing scales of Pierid butterflies. Proc Biol Sci 2007;274:359-66.

[20] Cromartie RIT. Insect pigments. Annu Rev Entomol 1959;4:59-76.

[21] Koch PB, Behnecke B, Weigmann-Lenz M, Ffrench-Constant RH. Insect pigmentation: activities of beta-alanyldopamine synthase in wing color patterns of wild-type and melanic mutant swallowtail butterfly Papilio glaucus. Pigment Cell Res 2000;13:54-8.

[22] Kato T, Sawada H, Yamamoto T, Mase K, Nakagoshi M. Pigment pattern formation in the quail mutant of the silkworm, Bombyx mori: parallel increase of pteridine biosynthesis and pigmentation of melanin and ommochromes. Pigment Cell Res 2006;19:337-45.

[23] Kopp A, Graze RM, Xu SZ, Carroll SB, Nuzhdin SV. Quantitative trait loci responsible for variation in sexually dimorphic traits in Drosophila melanogaster. Genetics 2003;163:771-87.

[24] Brisson JA, Templeton AR, Duncan I. Population genetics of the developmental gene optomotor-blind (omb) in Drosophila polymorpha: evidence for a role in abdominal pigmentation variation. Genetics 2004;168:1999-2010.

[25] Pool JE, Aquadro CF. The genetic basis of adaptive pigmentation variation in Drosophila melanogaster. Mol Ecol 2007;16:2844-51.

[26] Wittkopp PJ, Williams BL, Selegue JE, Carroll SB. Drosophila pigmentation evolution: divergent genotypes underlying convergent phenotypes. Proc Natl Acad Sci U S A 2003;100:1808-13.

[27] Llopart A, Comeron JM, Brunet FG, Lachaise D, Long M. Intron presence-absence polymorphism in Drosophila driven by positive Darwinian selection. Proc Natl Acad Sci U S A 2002;99:8121-6.

[28] Kopp A, Duncan I, Carroll SB. Genetic control and evolution of sexually dimorphic characters in Drosophila. Nature 2000;408:553-9.

[29] Wittkopp PJ, Vaccaro K, Carroll SB. Evolution of yellow gene regulation and pigmentation in Drosophila. Curr Biol 2002;12:1547-56.

[30] Brunetti CR, Selegue JE, Monteiro A, French V, Brakefield PM, Carroll SB. The generation and diversification of butterfly eyespot color patterns. Curr Biol 2001;11:1578-85.

[31] Beldade P, Brakefield PM, Long AD. Contribution of Distal-less to quantitative variation in butterfly eyespots. Nature 2002;415:315-8.

[32] Kronforst MR, Young LG, Kapan DD, McNeely C, O’Neill RJ, Gilbert LE. Linkage of butterfly mate preference and wing color preference cue at the genomic location of wingless. Proc Natl Acad Sci U S A 2006; 103:6575-80.

[33] Clark R, Brown SM, Collins SC, Jiggins CD, Heckel DG, Vogler AP. Colour pattern specification in the Mocker swallowtail Papilio dardanus: the transcription factor invected is a candidate for the mimicry locus $\mathrm{H}$. Proc Biol Sci 2008;275:1181-8.

[34] Reed RD, McMillan WO, Nagy LM. Gene expression underlying adaptive variation in Heliconius wing patterns: non-modular regulation of overlapping cinnabar and vermilion prepatterns. Proc Biol Sci 2008;275:37-45.

[35] Futahashi R, Fujiwara H. Melanin-synthesis enzymes coregulate stage-specific larval cuticular markings in the swallowtail butterfly, Papilio xuthus. Dev Genes Evol 2005;215:519-29.

[36] Futahashi R, Fujiwara H. Expression of one isoform of GTP cyclohydrolase coincides with the larval black markings of the swallowtail butterfly, Papilio xuthus. Insect Biochem Mol Biol 2006;36:63-70.

[37] Futahashi R, Fujiwara H. Regulation of 20-hydroxyecdysone on the larva pigmentation and the expression of melanin synthesis enzymes and yellow gene of the swallowtail butterfly, Papilio xuthus. Insect Biochem Mol Biol 2007;37:855-64.

[38] Gomulski LM, Pitts RJ, Costa S, et al. Genomic organization and characterization of the white locus of the Mediterranean fruitfly, Ceratitis capitata. Genetics 2001;157:1245-55.

[39] Albert S, Klaudiny J. The MRJP/YELLOW protein family of Apis mellifera: identification of new members in the EST library. J Insect Physiol 2004;50:51-9.

[40] Xia AH, Zhou QX, Yu LL, et al. Identification and analysis of YELLOW protein family genes in the silkworm, Bombyx mori. BMC Genomics 2006;7:195.

[41] Komoto N. A deleted portion of one of the two xanthine dehydrogenase genes causes translucent larval skin in the oq mutant of the silkworm (Bombyx mori). Insect Biochem Mol Biol 2002;32:591-7.

[42] Lorenzen MD, Brown SJ, Denell RE, Beeman RW. Cloning and characterization of the Tribolium castaneum eye-color genes encoding tryptophan oxygenase and kynurenine 3-monooxygenase. Genetics 2002;160:225-34.

[43] Haag ES, True JR. Perspective: From mutants to mechanisms? Assessing the candidate gene paradigm in evolutionary biology. Evolution Int J Org Evolution 2001;55:1077-84.

[44] Simpson P. The stars and stripes of animal bodies: evolution of regulatory elements mediating pigment and bristle patterns in Drosophila. Trends Genet 2007;23:350-8.

[45] Wray GA. The evolutionary significance of cis-regulatory mutations. Nat Rev Genet 2007;8:206-16. 
[46] Carroll SB. Evo-devo and an expanding evolutionary synthesis: a genetic theory of morphological evolution. Cell 2008;134:25-36.

[47] Stern DL, Orgogozo V. The loci of evolution: how predictable is genetic evolution? Evolution 2008;62:2155-77.

[48] Sword GA, Simpson SJ, El Hadi OT, Wilps H. Density-dependent aposematism in the desert locust. Proc Biol Sci 2000;267:63-8.

[49] Forsman A, Ringblom K, Civantos E, Ahnesjo J. Coevolution of color pattern and thermoregulatory behavior in polymorphic pygmy grasshoppers Tetrix undulata. Evolution 2002;56:349-60.

[50] Tibbetts EA, Dale J. A socially enforced signal of quality in a paper wasp. Nature 2004;432:218-22.

[51] Yeh SD, Liou SR, True JR. Genetics of divergence in male wing pigmentation and courtship behavior between Drosophila elegans and D. gunungcola. Heredity 2006;96:383-95.

[52] Leo Lester R, Grach C, Paul Pener M, Simpson SJ. Stimuli inducing gregarious colouration and behaviour in nymphs of Schistocerca gregaria. J Insect Physiol 2005;51:737-47.

[53] Simpson SJ, Miller GA. Maternal effects on phase characteristics in the desert locust, Schistocerca gregaria: a review of current understanding. J Insect Physiol 2007:53:869-76.

[54] Wilson RJ, Goodman JL, Strelets VB. FlyBase: integration and improvements to query tools. Nucleic Acids Res 2008;36:D588-93.

[55] Beall CJ, Hirsh J. Regulation of the Drosophila dopa decarboxylase gene in neuronal and glial cells. Genes Dev 1987;1:510-20.

[56] Drapeau MD, Radovic A, Wittkopp PJ, Long AD. A gene necessary for normal male courtship, yellow, acts downstream of fruitless in the Drosophila melanogaster larval brain. J Neurobiol 2003;55:53-72.

[57] Wagner S, Heseding C, Szlachta K, True JR, Prinz H, Hovemann BT. Drosophila photoreceptors express cysteine peptidase tan. J Comp Neurol 2007;500:601-11.

[58] True JR, Yeh SD, Hovemann BT, et al. Drosophila tan encodes a novel hydrolase required in pigmentation and vision. PLoS Genet 2005;1:e63.

[59] Hodgetts RB, O'Keefe SL. Dopa decarboxylase: a model gene-enzyme system for studying development, behavior, and systematics. Annu Rev Entomol 2006;51:259-84

[60] Drapeau MD, Cyran SA, Viering MM, Geyer PK, Long AD. A cis-regulatory sequence within the yellow locus of Drosophila melanogaster required for normal male mating success. Genetics 2006;172:1009-30.

[61] Sawada H, Nakagoshi M, Mase K, Yamamoto T. Occurrence of ommochromecontaining pigment granules in the central nervous system of the silkworm, Bombyx mori. Comp Biochem Physiol B Biochem Mol Biol 2000;125:421-8.

[62] Nappi AJ, Christensen BM. Melanogenesis and associated cytotoxic reactions: applications to insect innate immunity. Insect Biochem Mol 2005;35:443-59.

[63] Barnes AI, Siva-Jothy MT. Density-dependent prophylaxis in the mealworm beetle Tenebrio molitor L. (Coleoptera: Tenebrionidae): cuticular melanization is an indicator of investment in immunity. Proc Biol Sci 2000;267:177-82.

[64] Galko MJ, Krasnow MA. Cellular and genetic analysis of wound healing in Drosophila larvae. PLoS Biol 2004;2:1114-26.

[65] Brakefield PM, French V. Eyespot development on butterfly wings: the epidermal response to damage. Dev Biol 1995;168:98-111.

[66] Kramer KJ, Hopkins TL. Tyrosine metabolism for insect cuticle tanning. Arch Insect Biochem Physiol 1987;4:279-301.

[67] Nappi AJ, Vass E. Melanogenesis and the generation of cytotoxic molecules during insect cellular immune reactions. Pigment Cell Res 1993;6:117-26.

[68] Dombeck I, Jaenike J. Ecological genetics of abdominal pigmentation in Drosophila falleni: a pleiotropic link to nematode parasitism. Evolution 2004;58:587-96.

[69] Wilson K, Cotter SC, Reeson AF, Pell JK. Melanism and disease resistance in insects. Ecol Lett 2001;4:637-49.

[70] Gorman MJ, An C, Kanost MR. Characterization of tyrosine hydroxylase from Manduca sexta. Insect Biochem Mol Biol 2007;37:1327-37.

[71] Sideri M, Tsakas S, Markoutsa E, Lampropoulou M, Marmaras VJ. Innate immunity in insects: surface-associated dopa decarboxylase-dependent pathways regulate phagocytosis, nodulation and melanization in medfly haemocytes. Immunology 2008;123:528-37.

[72] Marmaras VJ, Charalambidis ND, Zervas CG. Immune response in insects: the role of phenoloxidase in defense reactions in relation to melanization and sclerotization. Arch Insect Biochem Physiol 1996;31:119-33.
[73] Sugumaran M. Comparative biochemistry of eumelanogenesis and the protective roles of phenoloxidase and melanin in insects. Pigment Cell Res 2002;15:2-9

[74] Mavrouli MD, Tsakas S, Theodorou GL, Lampropoulou M, Marmaras VJ. MAP kinases mediate phagocytosis and melanization via prophenoloxidase activation in medfly hemocytes. Biochim Biophys Acta 2005;1744:145-56.

[75] Ling E, Yu XQ. Prophenoloxidase binds to the surface of hemocytes and is involved in hemocyte melanization in Manduca sexta. Insect Biochem Mol Biol 2005;35:1356-66.

[76] Kalmus $\mathrm{H}$. The resistance to desiccation of Drosophila mutants affecting body colour. Proc Roy Soc London B 1941;130:185-201.

[77] Rajpurohit S, Parkash R, Niwas SR, Nedved O, Singh S. Parallel trend in pigmentation and desiccation tolerance: altitudinal and latitudinal effects in Drosophila melanogaster. Dros Inf Serv 2007;90:70-9.

[78] Brisson JA, De Toni DC, Duncan I, Templeton AR. Abdominal pigmentation variation in Drosophila polymorpha: geographic variation in the trait, and underlying phylogeography. Evolution 2005;59:1046-59.

[79] Norgate M, Lee E, Southon A, et al. Essential roles in development and pigmentation for the Drosophila copper transporter DmATP7. Mol Biol Cell 2006; 17:475-84.

[80] Hsouna A, Lawal HO, Izevbaye I, Hsu T, O’Donnell JM. Drosophila dopamine synthesis pathway genes regulate tracheal morphogenesis. Dev Biol 2007;308:30-43.

[81] De Luca M, Roshina NV, Geiger-Thornsberry GL, Lyman RF, Pasyukova EG, Mackay TF. Dopa decarboxylase (Ddc) affects variation in Drosophila longevity. Nat Genet 2003;34:429-33.

[82] Carbone MA, Jordan KW, Lyman RF, et al. Phenotypic variation and natural selection at catsup, a pleiotropic quantitative trait gene in Drosophila. Curr Biol 2006;16:912-9.

[83] Simonsen A, Cumming RC, Lindmo K, et al. Genetic modifiers of the Drosophila blue cheese gene link defects in lysosomal transport with decreased life span and altered ubiquitinated-protein profiles. Genetics 2007;176:1283-97.

[84] Wagner GP, Lynch VJ. The gene regulatory logic of transcription factor evolution. Trends Ecol Evol 2008;23:377-85.

[85] Langley CH, Lazzaro BP, Phillips W, Heikkinen E, Braverman JM. Linkage disequilibria and the site frequency spectra in the su(s) and $\mathrm{su}(\mathrm{w}(\mathrm{a}))$ regions of the Drosophila melanogaster X chromosome. Genetics 2000;156:1837-52.

[86] Wagner GP, Kenney-Hunt JP, Pavlicev M, Peck JR, Waxman D, Cheverud JM. Pleiotropic scaling of gene effects and the 'cost of complexity'. Nature 2008;452:470-2.

[87] de Meaux J, Pop A, Mitchell-Olds T. Cis-regulatory evolution of chalconesynthase expression in the genus Arabidopsis. Genetics 2006;174: 2181-202.

[88] McGregor AP, Orgogozo V, Delon I, et al. Morphological evolution through multiple cis-regulatory mutations at a single gene. Nature 2007;448:587-90.

[89] Miller CT, Beleza S, Pollen AA, et al. cis-Regulatory changes in Kit ligand expression and parallel evolution of pigmentation in sticklebacks and humans. Cell 2007;131:1179-89.

[90] Mullen LM, Hoekstra HE. Natural selection along an environmental gradient: a classic cline in mouse pigmentation. Evolution 2008;62:1555-70.

[91] Mundy NI. A window on the genetics of evolution: MC1R and plumage colouration in birds. Proc Biol Sci 2005;272:1633-40.

[92] Canestro C, Yokoi H, Postlethwait JH. Evolutionary developmental biology and genomics. Nat Rev Genet 2007;8:932-42.

[93] Mitchell-Olds T, Feder M, Wray G. Evolutionary and ecological functional genomics. Heredity 2008;100:101-2.

[94] Abzhanov A, Extavour CG, Groover A, et al. Are we there yet? Tracking the development of new model systems. Trends Genet 2008;24:353-60.

[95] Stern DL. The developmental genetics of microevolution. Novartis Found Symp 2007;284:191-200.

[96] Nijhout HF. Development and evolution of adaptive polyphenisms. Evol Dev 2003;5:9-18.

[97] Gibert JM, Peronnet F, Schlötterer C. Phenotypic plasticity in Drosophila pigmentation caused by temperature sensitivity of a chromatin regulator network. PLoS Genet 2007;3:e30.

[98] Jeong S, Rokas A, Carroll SB. Regulation of body pigmentation by the Abdominal-B Hox protein and its gain and loss in Drosophila evolution. Cell 2006;125:1387-99. 